Porfírio Candanedo GUERRA $^{1}$

Luiz Carlos VULCANO²

Noeme de Sousa ROCHA ${ }^{3}$

Correspondência para: PORFÍRIOCANDANEDOGUERRA

Departamento das Clínicas

Curso de Medicina Veterinária

Centro de Ciências Agrárias

Universidade Estadual do Maranhão

Rua dos Professores, s/n-Bl. A2, apto. 304

- Bairro Cohafuma

65078-300 São Luis - Maranhão.

totipc@hotmail.com

Recebido para publicação: 17/05/2004 Aprovado para publicação: 13/02/2006

\title{
Avaliação radiográfica, densitométrica e histológica do uso de perfurações ósseas para o estímulo de consolidação de fraturas do terço distal do rádio de cães
}

\author{
1 - Departamento das Clínicas do Curso de Medicina Veterinária do Centro \\ de Ciências Agrárias da Universidade Estadual do Maranhão, São Luis - MA \\ 2 - Departamento de Reprodução Animal e Radiologia Veterinária da \\ Faculdade de Medicina Veterinária e Zootecnia da Universidade Estadual \\ Paulista Julio de Mesquita, Botucatu - SP \\ 3 - Departamento de Clínica Veterinária da Faculdade de Medicina \\ Veterinária e Zootecnia da Universidade Estadual Paulista Julio de Mesquita; \\ Botucatu - SP
}

\begin{abstract}
Resumo
Objetivando estimular o processo de formação do calo ósseo do terço distal do rádio de cães de pequeno porte, foram utilizados 24 animais srd, adultos, de ambos os sexos. Os cães, separados em dois grupos experimentais de 12 animais denominados de controle e tratado foram divididos em 4 momentos $(\mathrm{m} 1=15$ dias, $\mathrm{m} 2=30$ dias, $\mathrm{m} 3=45$ dias e $\mathrm{m} 4=60$ dias), e submetidos a fraturas cirúrgicas. no grupo tratado, foram realizadas perfurações ósseas nos bordos do foco da fratura, no sentido crânio-caudal e médio-lateral. No final de cada momento, os animais controles e tratados foram avaliados radiograficamente, histologicamente e a densidade mineral óssea (dmo) determinada no local da fratura. $\mathrm{Na}$ avaliação radiográfica do grupo tratado com 15 e 30 dias foi verificado o processo de reparação óssea mais intenso do que no grupo controle. Nos momentos m3 e $\mathrm{m} 4$, não se observaram diferenças no processo de reparação entre os dois grupos. Os valores da dmo foram maiores nos animais tratados. o estudo histológico revelou aos 15 e 30 dias, hiperplasia de condrócitos, e inicio de ossificação endocondral nos membros perfurados; o grupo controle mostrou tecido conjuntivo de sustentação e início de hiperplasia de condrócitos. Nos m3 e m4 do grupo tratado, se verificaram formação e remodelação de calo periosteal em fases mais avançadas quando comparados ao grupo controle. conclui-se que o uso de perfurações melhora o suprimento de fluxo sangüíneo e a ativação de células osteogênicas no local de fratura, estimulando o início do processo de consolidação da fratura.
\end{abstract}

\section{Introdução}

A ocorrência de fraturas que comprometem a região média e distal dos ossos rádio e ulna em cães de pequeno porte é comum, e em muitas delas é freqüente a união retardada ou a não união. Estas complicações são o resultado, dentre outras causas, de um suprimento sangüíneo deficiente, falhas na estabilização da fratura
Palavras-chave: Fratura.

Perfuração óssea. Densitometria. Histologia. Cão. e pouco tecido mole local $1^{1,2,3,4,5,6,7,8}$. Diversas técnicas têm sido utilizadas para reduzir esse problema e devolver rapidamente as funções ao membro, porém apresentam contraindicações e resultados insatisfatórios o que tem impedido a obtenção do sucesso ${ }^{3,8}$. Problemas de cicatrização de fratura vêm sendo objeto de estudo desde o início do século passado. a realização de orifícios em focos de fratura de tíbias de humanos, como forma de induzir a união das bordas dos fragmentos 
ósseos já foi feita?. o uso dessa técnica em fratura incompleta de fíbula resultou em uma rápida resolução, já que de acordo com estudos, a mesma provoca alterações na hemodinâmica local ${ }^{10,11}$. O acompanhamento da consolidação de uma fratura usando métodos não invasivos, como densitometria, tomografia e ressonância magnética, possibilita detectar alterações que ocorrem ainda em fases iniciais do processo ${ }^{12}$. A avaliação histológica da reparação de uma fratura permite acompanhar e definir especificamente todas as etapas que ocorrem na consolidação, além de identificar fatores que interferem na reparação adequada ${ }^{13}$.

A utilização de técnicas densitométricas constitui uma importante ferramenta no estudo do esqueleto ${ }^{14}$. A determinação da densidade mineral óssea é parte integral do diagnóstico, planejamento terapêutico e monitorização de indivíduos com doenças ósseas. Para uma avaliação da mineralização ou da densidade mineral óssea (dmo) dos animais, torna-se necessário o emprego de técnicas de medidas in vivo ${ }^{15}$; dentre elas se incluem a absorção direta por fótons ${ }^{16}$, análise de ativação por nêutrons ${ }^{17}$, tomografia computadorizada ${ }^{18}$, fotometria radiográfica $^{19}$ e a densitometria óptica em imagem radiográfica ${ }^{20}$, sendo que esta última pode ser utilizada para uma análise seqüencial da massa óssea, com um custo menor que as demais metodologias e tendo como fator preponderante a precisão ${ }^{20,21,22}$.

A densitometria óptica em imagens radiográficas foi utilizada para avaliar as densidades ósseas em ulnas de coelhos, após o uso de perfurações. Os valores densitométricos obtidos foram confrontados com o material histológico retirado da área de perfuração. no experimento, o preenchimento ósseo dos orifícios foi estimulado por meio de ultrasom pulsatil. quanto à avaliação do tratamento com esse aparelho, a técnica densitométrica não mostrou diferença significativa entre os membros tratados e controles, assim mesmo, as observações histológicas relatam presença de tecido conjuntivo fibroso cartilaginoso com formação de trabéculas ósseas nos dois grupos. Este método não invasivo fornece informações equivalentes às conseguidas pela análise histológica, demonstrando ser altamente eficaz e promissor ${ }^{23}$. estudo da cicatrização do tecido ósseo de fraturas em rádio e ulna de cães adultos, através de microangiografias demonstrou que o sistema arterial medular é mais importante que o periosteal na vascularização da camada cortical diafisária em pelo menos dois terços da estrutura do osso; evidenciaram ainda a presença de tecido cartilaginoso (conjuntivo e neoformação óssea) somente na linha da fratura $^{24}$. Pesquisas para investigar o efeito da somatotropina canina recombinante, no metabolismo e aspectos histológicos do processo de consolidação de fraturas em cães foram realizadas. O estudo histológico realizado na oitava semana do experimento demonstrou que, o grupo tratado apresentava atividade osteogênica maior, com evidência de ossificação endocondral no calo. No outro grupo o local da fratura mostrava tecido fibroso orientado perpendicularmente ao longo do axis do osso. Verificaram-se ainda regiões de fibrocartilagem sem evidência de ossificação. De acordo com os resultados, o uso do hormônio aumentou a atividade osteogênica favorecendo o processo de reparação no local da fratura ${ }^{25}$.

\section{Materiais e Métodos}

Foram utilizados 24 cães SRD, machos e fêmeas, com idades entre 1 e 3,6 anos que apresentavam o eixo ósseo do rádio delgado, provenientes do Biotério Central do Campus de Botucatu, pesando entre $4 \mathrm{e}$ $8 \mathrm{~kg}$. Os animais foram mantidos em canis de experimentação do Hospital Veterinário da Faculdade de Medicina Veterinária e Zootecnia da Universidade Estadual Paulista - Campus de Botucatu, recebendo tratamento antiparasitário e profilático de moléstias infecciosas, alimentados com ração e água ad libitum e mantidos em iguais condições de manejo.

Os animais foram separados em dois 
grupos experimentais de 12 cães cada; tratados e controles. Cada grupo correspondeu a quatro momentos de estudo: M1 (15 dias após cirurgia) M2 (30 dias), M3 (45 dias) e M4 (60 dias). Em cada momento se utilizaram seis animais, três para cada grupo. Após anestesia ${ }^{8,15}$, nos animais controles e tratados de cada momento foram provocadas fraturas transversais ao eixo do osso, no terço distal do rádio direito, usando osteótomo e martelo. Nos tratados, perfurações perpendiculares de $1 \mathrm{~mm}$ no sentido crânio-caudal e médio-lateral foram realizadas nas extremidades dos fragmentos fraturados. A imobilização externa foi feita com tala de cloreto de polivinila (PVC).

Radiografias foram realizadas antes do procedimento cirúrgico (MI), para avaliar a integridade óssea e determinar os valores da densidade mineral óssea (DMO) da região distal do radio. Avaliações radiográficas, densitométricas e histológicas foram feitas no foco da fratura em M1, M2, M3, e M4 nos animais de ambos os grupos.
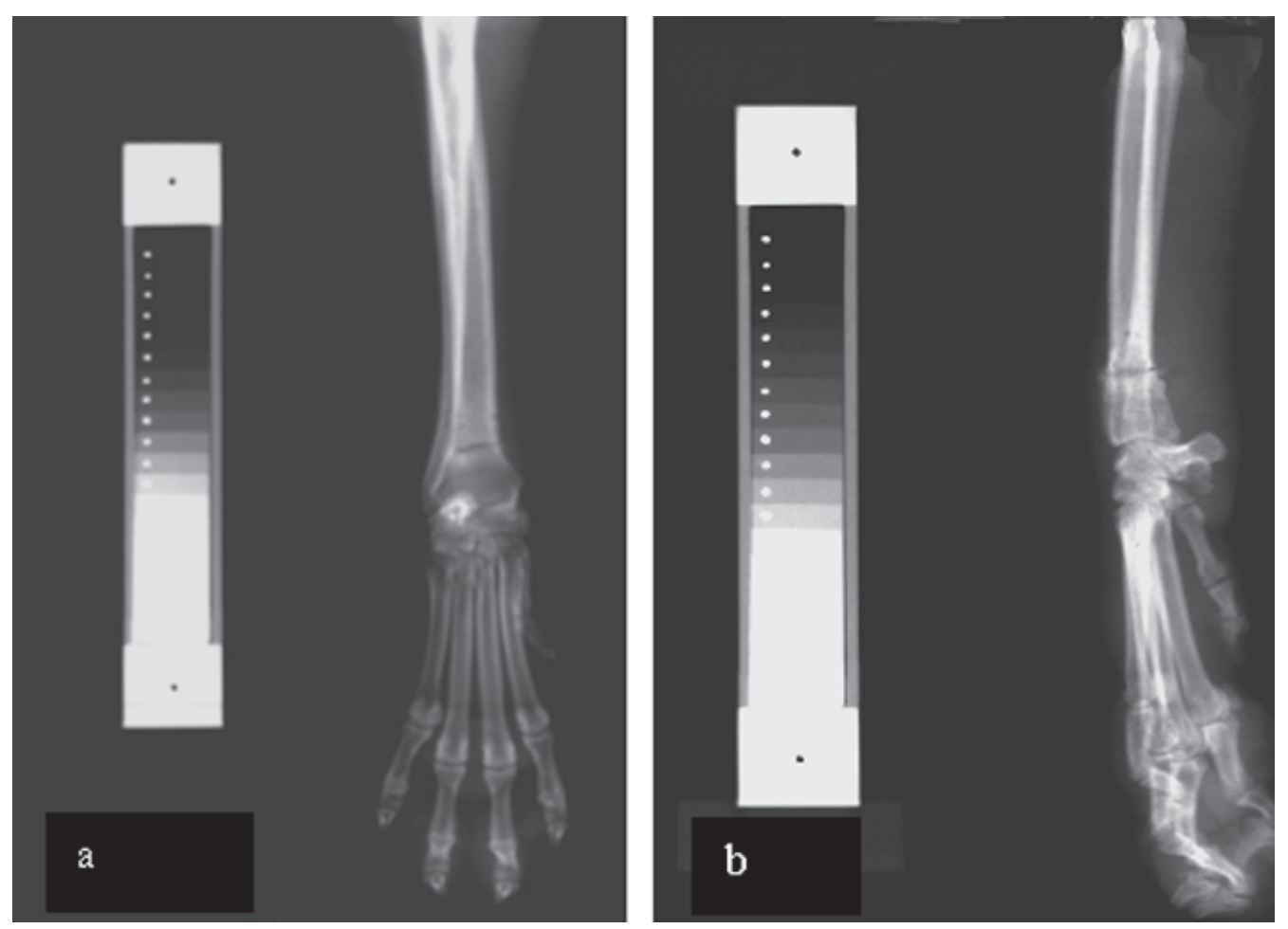

Figura 1 - Imagem radiográfica de fratura na extremidade distal do rádio em projeções (a) crânio-caudal e (b) médio-lateral do membro torácico direito, grupo do tratado aos 15 dias pós-operatório. Observar-se reação periosteal leve, bordas com formação de ponte óssea e aumento de densidade em fenda da fratura. Botucatu - 2002
Foi utilizado o método de densitométria óptica em imagens radiográficas para avaliação da densidade mineral nas extremidades do foco da fratura ${ }^{11}$ e as leituras seguiram o protocolo estabelecido para cães ${ }^{10}$.

Para realizar o estudo histológico, foram feitas eutanásia nos animais tratados e controle (aprovação protoc. 025/99-CEEA, UnespBotucatu em 11/02/2000), e os ossos fraturados experimentalmente, retirados e fixados em formalina tamponada a $10 \%$ sendo posteriormente submetidos ao processo de descalcificação ${ }^{17}$. A seguir, foram preparadas lâminas com cortes histológicos de $4 \mathrm{~mm}$ do tecido ósseo localizado no foco da fratura, em seguida se procedeu às colorações rotineiras de Hematoxilina -Eosinae Alcian Pounceu ${ }^{3}$. A leitura foi feita em microscópio óptico.

\section{Resultados}

No estudo radiográfico do primeiro 


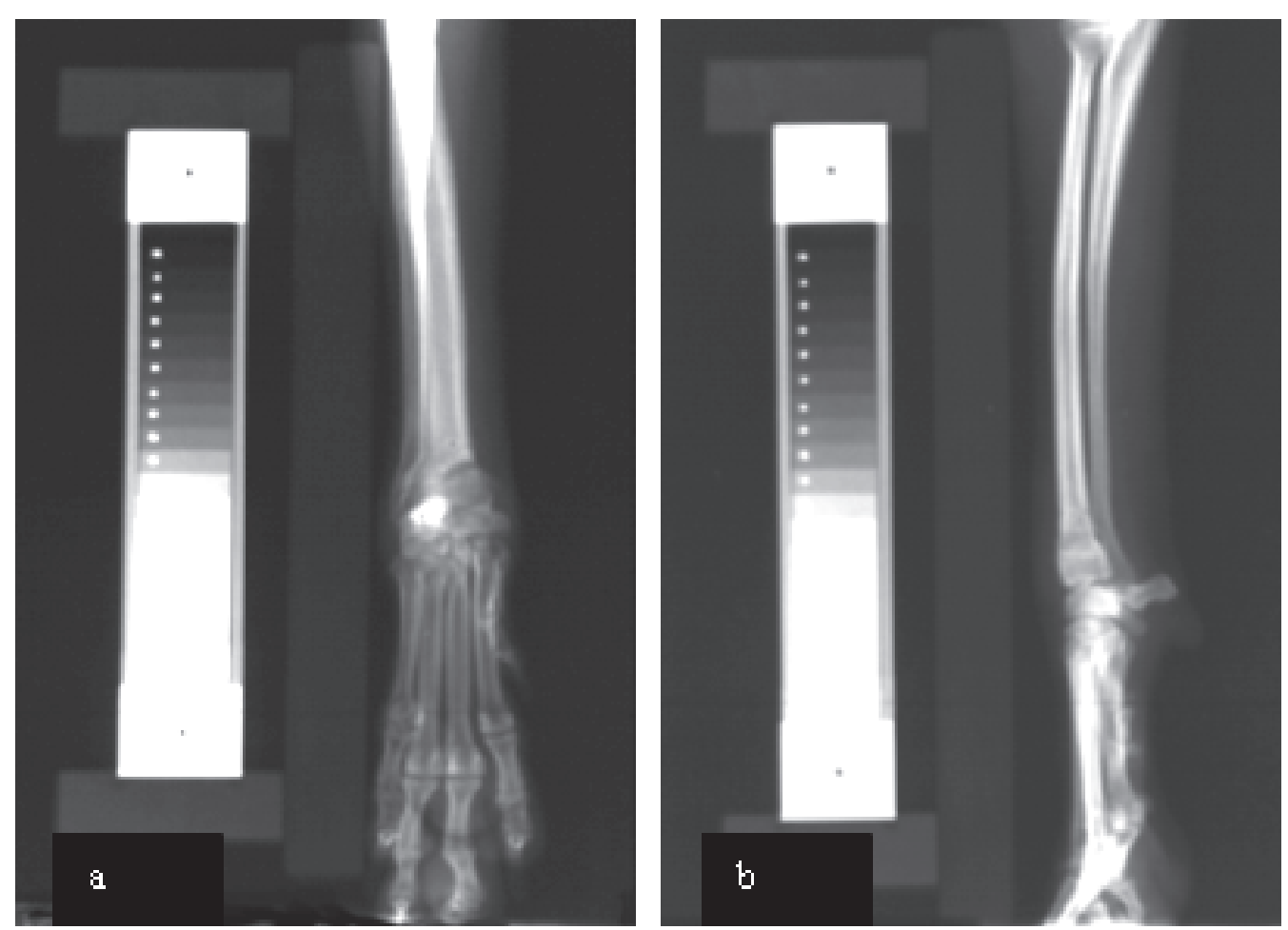

Figura 2 - Imagem radiográica de fratura na extremidade distal do rádio em projeções (a) crânio-caudal e (b) médio-lateral do membro torácico direito, grupo tratado aos 30 dias pós-operatório. Observar-se união de bordas em fase lateral e calo ósseo em formação no foco da fratura.Botucatu - 2002

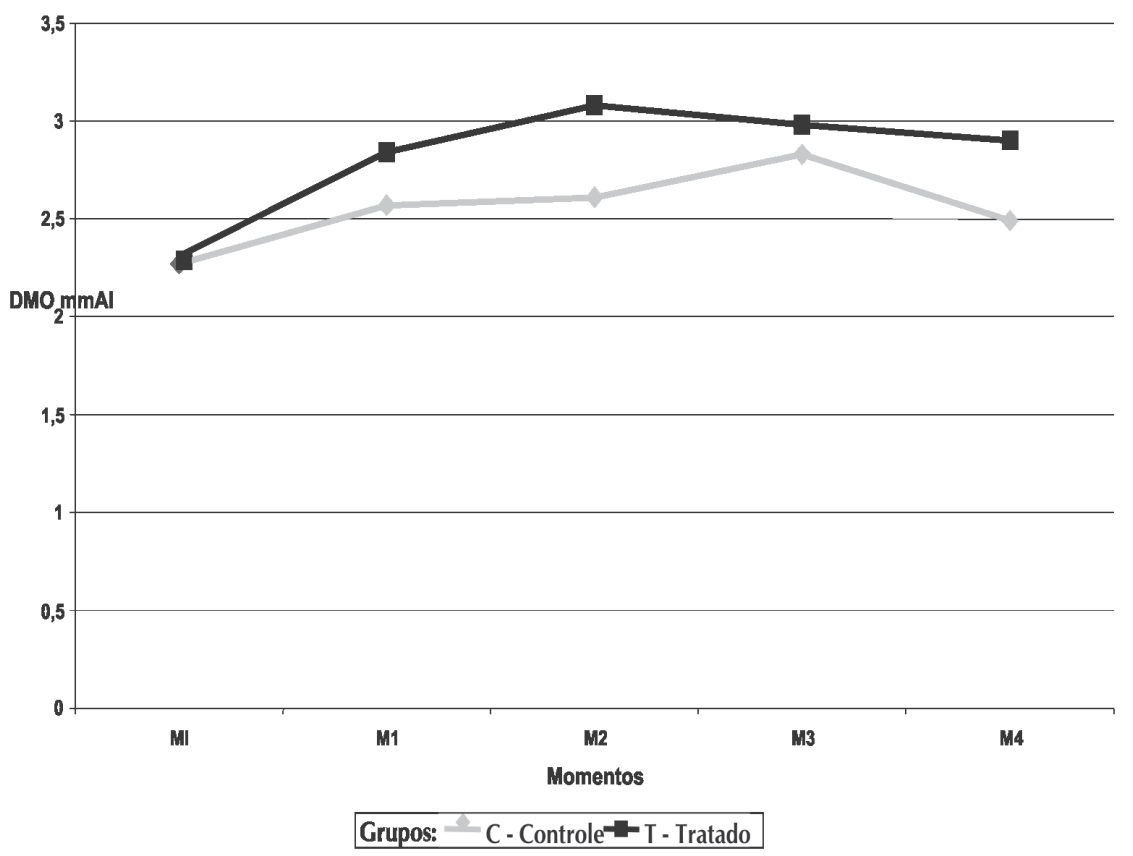

Figura 2 - Valores médios da Densidade Mineral Óssea (DMO) em milímetros de alumínio (mmAl) do local da fratura no terço distal do rádio de 24 cães, grupos controles e tratados nos cincos momentos estudados. Botucatu - 2002 
Figura 3 - Valores que deram origem ao gráfico

\begin{tabular}{|c|c|c|c|c|c|c|c|c|c|c|}
\hline \multicolumn{11}{|c|}{ Momentos } \\
\hline & $\mathrm{Ml}$ & & M1 & & M2 & & M3 & & M4 & \\
\hline $\mathrm{N}^{\circ}$ & C & $\mathrm{T}$ & $\mathrm{C}$ & $\mathrm{T}$ & C & $\mathrm{T}$ & C & $\mathrm{T}$ & $\mathrm{C}$ & $\mathrm{T}$ \\
\hline 01 & 1,80 & 2,59 & 2,00 & 2,88 & & & & & & \\
\hline 02 & 2,47 & 2,00 & 3,18 & 2,49 & & & & & & \\
\hline 03 & 2,45 & 2,16 & 2,52 & 2,42 & & & & & & \\
\hline 04 & 2,08 & 2,12 & 2,81 & 3,11 & 2,00 & 3,79 & & & & \\
\hline 05 & 2,08 & 2,22 & 2,55 & 3,08 & 2,60 & 3,37 & & & & \\
\hline 06 & 2,00 & 2,69 & 2,04 & 3,45 & 1,80 & 1,90 & & & & \\
\hline 07 & 2,47 & 2,15 & 2,66 & 2,98 & 2,87 & 2,89 & 2,96 & 3,03 & & \\
\hline 08 & 2,25 & 2,20 & 2,33 & 2,55 & 3,24 & 3,31 & 2,87 & 3,23 & & \\
\hline 09 & 2,42 & 2,09 & 3,01 & 2,73 & 3,06 & 3,62 & 2,80 & 2,80 & & \\
\hline 10 & 2,30 & 2,14 & 2,52 & 2,77 & 2,54 & 2,89 & 2,78 & 2,94 & 2,35 & 2,91 \\
\hline 11 & 2,40 & 2,70 & 2,54 & 2,85 & 2,64 & 3,07 & 2,83 & 2,98 & 2,49 & 2,91 \\
\hline 12 & 2,62 & 2,75 & 2,73 & 2,86 & 2,73 & 2,90 & 2,75 & 2,90 & 2,63 & 2,90 \\
\hline$x$ & 2,27 & 2,31 & 2,57 & 2,84 & 2,61 & 3,08 & 2,83 & 2,98 & 2,49 & 2,90 \\
\hline$S$ & 0,24 & 0,27 & 0,34 & 0,29 & 0,46 & 0,55 & 0,07 & 0,14 & 0,14 & 0,05 \\
\hline
\end{tabular}

MI - (momento inicial), M1 - (15 dias), M2 - (30 dias), M3 - (45 dias), M4 - (60 dias).

C-grupo controle

$\mathrm{T}$ - grupo tratado

momento (M1) observou-se que os focos das fraturas no grupo controle, em sua maioria não mostravam reação periosteal, notou-se ainda, densidade aumentada na fenda e bordas da fratura na maioria das amostras. No grupo tratado, verificou-se que as perfurações realizadas no local da fratura promoveram reação periosteal discreta a moderada, fato esse observado na maioria dos animais. Notou-se também, nas bordas das fraturas, formação de ponte óssea, aumento de densidade e na maioria dos membros, o eixo ósseo alinhado. Os orifícios foram visualizados como áreas radiolucentes, próximos aos extremos dos fragmentos fraturados (Figura1). No M2 do grupo controle, observou-se reação periosteal em todos os focos de fraturas, algumas bordas de fratura mostrando formação de ponte óssea e a maioria dos eixos ósseos alinhados. No grupo tratado, observou-se, além de reação periosteal em alguns focos de fratura, formação de ponte e calo ósseo e em alguns, reparação bastante avançada. Os orifícios próximos às bordas das fraturas mostraram- se como áreas densas (Figura.2). Nos M3 e M4 os dois grupos mostraram adiantado estágio de consolidação de fratura, porém a linha de fratura ainda era visível.

A densidade mineral óssea (DMO) dos membros torácicos antes da cirurgia (MI) dos grupos controle $(2,27 \pm 0,24$ mmAl) e tratado $(2,31 \pm 0,27 \mathrm{mmAl})$ apresentaram valores absolutos próximos. Nos M1 e M2 do grupo controle (2,57 \pm $0,34$ e 2,61 $\pm 0,46)$, constatou-se aumento de valores, evento esse também observado nos mesmos momentos do grupo tratado $(2,84 \pm 0,29$ e $3,08 \pm 0,55)$, porém de forma mais acentuada. No terceiro momento do grupo controle, verifica-se que, enquanto os valores absolutos ainda apresentam aumento, $(2,83 \pm 0,07)$ o tratado começa a mostrar diminuição $(2,98 \pm 0,14)$. Tais valores demonstram que a DMO do calo ósseo do grupo tratado teve seu maior valor absoluto aos 30 dias pós-operatório, o mesmo ocorrendo no controle somente ao 45 dias. Observa-se também, que entre M3 e M4 do grupo controle $(2,83 \pm 0,07$ e $2,49 \pm$ 


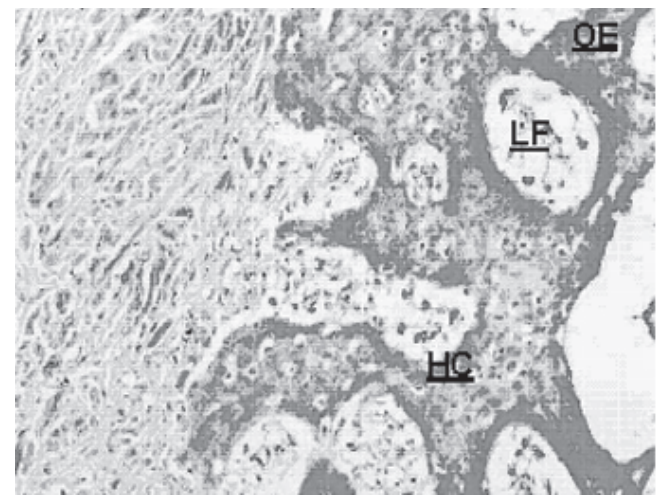

Figura 4 - Imagem histológica do local de fratura, na extremidade distal do rádio, grupo tratado aos 15 dias pós-operatório. Observam-se lacunas formadas (LF), hiperplasia de ondrócitos (HC) e ossificação endocondral (OE). Coloração Alcian Ponceau. (400X). Botucatu - 2002

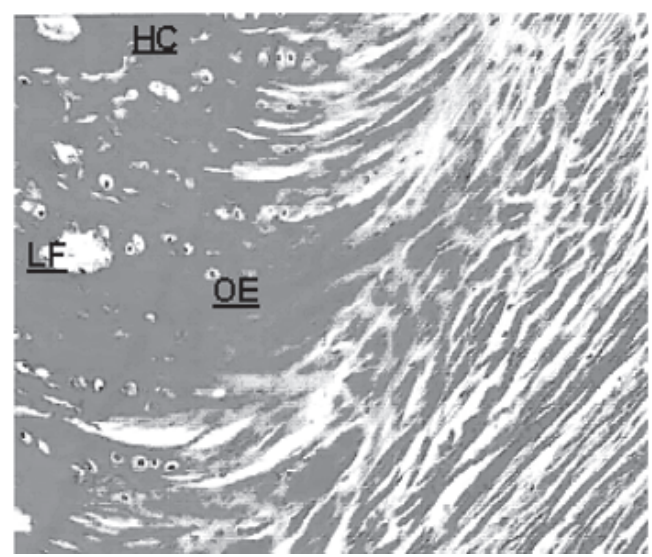

Figura 5 - Imagem histológica do local de fratura, na extremidade distal do rádio, grupo tratado aos 30 dias pós-operatório. Observam-se lacunas formadas (LF), hiperplasia de condrócitos (HC), ossificação endocondral (OE). Coloração Alcian Ponceau (400X). Botucatu - 2002

$0,14)$ e do tratado $(2,98 \pm 0,14$ e $2,90 \pm$ $0,05)$, ocorre uma diminuição de valores, sendo mais acentuada no controle. Os valores absolutos da DMO do grupo tratado estão acima dos obtido pelo grupo controle (Figura. 3).

O estudo histológico do foco da fratura do terço distal do rádio mostra que, aos 15 dias, os animais tratados mostraram hiperplasia de condrócitos em fase mais avançada, quando comparados com os controles. Nota-se ainda, ossificação endocondral em fase inicial, que não foi observada no grupo controle (Figura. 4). No

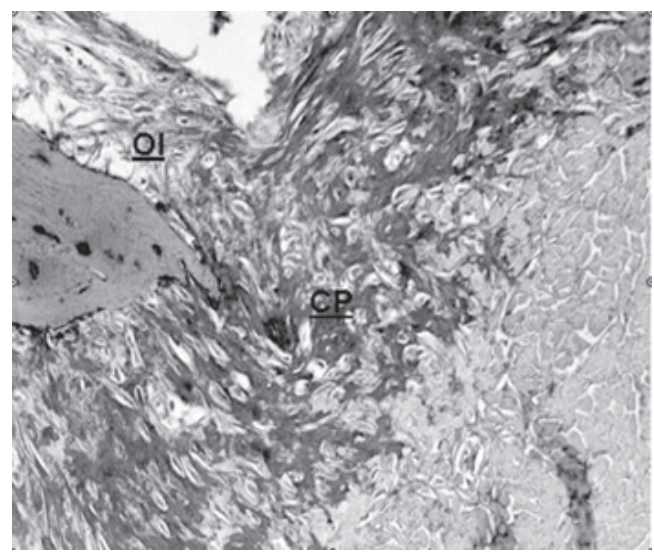

Figura 6-Imagem histológica do local da fratura, na extremidade distal do rádio, grupo tratado aos 45 dias pós-operatório. Observam-se osso imaturo (Ol) e formação de calo periosteal (CP). Coloração Alcian Ponceau (400). Botucatu-2002

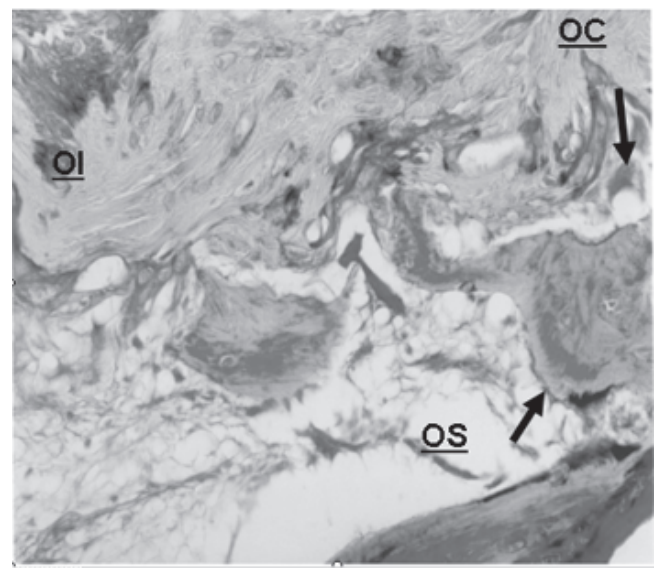

Figura 7 - Imagem histológica do local de fratura, na extremidade distal do rádio, grupo tratado aos 60 dias pós-operatório. Observam-se osso imaturo (OI), Osteoblastos (OS) e Osteoclastos (OC). Coloração. Alcian Ponceau (400X). Botucatu - 2002

M2, os focos das fraturas dos animais controles ainda mostraram hiperplasia de células cartilaginosas, enquanto o grupo tratado mostrou, além de uma ossificação endocondral avançada, maturação acentuada de lacunas. (Figura. 5). No terceiro momento, observou-se formação de um calo periosteal no foco da fratura dos animais tratados e presença de osso imaturo, já no controle os cortes histológicos mostraram áreas de hiperplasia de condrócitos e o início de ossificação endocondral (Figura. 6). No M4 
encontrou-se, no grupo tratado, além da formação do calo periosteal, o início da fase de remodelação óssea (Figura.7).

\section{Discussão}

Os resultados radiográficos, obtidos neste estudo, demonstraram que as reparações das fraturas, estimuladas por perfurações apresentaram início da atividade osteogênica já aos 15 dias, confirmando que o sistema vascular medular é mais eficiente na vascularização da camada cortical diafisária, uma vez que as perfurações permitem que fatores osteogênicos, como fluxo sangüíneo mais intenso no foco da fratura e células osteoprogenitoras, procedentes do periósteo, endósteo e medula óssea, se concentrem em maior quantidade no local da fratura, promovendo rápido início do processo de formação do calo ósseo ${ }^{5,11,15}$, como constatado em reparação de fraturas ósseas em humanos?.

A determinação da densidade mineral óssea (DMO) realizada no foco da fratura mostrou aumento dos valores absolutos logo aos 15 dias em ambos os grupos. Entretanto, o valor observado no grupo tratado foi superior $(2,84 \mathrm{mmAl} \pm 0,29)$ quando comparado com o controle $(2,57$ $\mathrm{mmAl} \pm 0,34)$, permitindo sugerir que a atividade osteogênica, que dá inicio ao processo de reparação óssea, tenha iniciado antes e com mais intensidade nos membros perfurados ${ }^{23}$. Ao avaliar o segundo momento do estudo, percebe-se que o valor obtido permanece elevado no grupo tratado, com diminuição da DMO em M3 e M4. No controle, entretanto, verifica-se aumento de valor até o M3 e posterior diminuição no último momento. Tais observações sugerem que a maior formação de massa óssea no foco da fratura do grupo tratado ocorreu no M2 e no controle no M3, e que o método, de acordo com pesquisadores, 20,22,26 pode ser utilizado para análise seqüencial de massa óssea.

Os resultados densitométricos, obtidos neste trabalho, quando comparados com os achados histológicos entre os grupos tratado e controle, evidenciaram que o uso de perfurações nas extremidades fraturadas promove reparação mais rápida do foco de fratura, especialmente nas fases iniciais (M1 e M2), diferente das observações comentadas por outros relatos ${ }^{21}$. Essa diferença pode estar relacionada com a espécie animal usada no estudo ou ainda com método utilizado como fator estimulador de formação óssea.

Os achados histológicos observados no estudo, como hiperplasia de condrócitos, ossificação endocondral, maturação acentuada de lacunas, formação e remodelação do calo periosteal, são semelhantes aos encontrados por outros autores ${ }^{5,25}$ ao trabalharem com outra substância bioativa. Porém, é importante destacar que, nesta pesquisa, a formação do calo periosteal foi percebida aos 45 dias (M3) de iniciado o experimento e na oitava semana (M4), já se observava o início de remodelação óssea, evento apenas verificados nas 12 semanas por pesquisadores que estudaram o processo de consolidação óssea, após fraturas cirúrgicas em cães de pequeno porte ${ }^{10}$. A presença desses achados em menor tempo pode ter sido influenciada pelo método utilizado para estimular a formação do calo no foco de fratura.

\section{Conclusões}

As perfurações ósseas, realizadas no foco de fratura do terço distal do rádio de cães, estimularam a osteogênese aos 15 dias de pós -operatório, provocando rápido início do processo de consolidação de fratura, evento esse não verificado no grupo controle.

O estudo densitométrico realizado no local da fratura mostrou que os valores absolutos da DMO em milímetro de alumínio do grupo tratado estão acima do obtido pelo grupo controle em todos os momentos.

A avaliação histológica do grupo tratado aos 15 e 30 dias de pós-operatório, 
mostrou o desenvolvimento de hiperplasia de condrócitos, formação de lacunas e ossificação endocondral em fases mais avançadas, quando comparado ao grupo controle.

\section{Radiographic, densitometric and histological evaluation of the use of bone perforations on fracture consolidation of distal third of radius from dogs.}

\section{Abstract}

To stimulate the bone callus development process from distal third of radius, 24 adult mongrel dogs used were from both sexes. These dogs were separated in two experimental groups of 12 animals each, named control and treated, divided in 4 moments $(M 1=15$ days; $\mathrm{M} 2=30$ days; $\mathrm{M} 3=45$ days; $\mathrm{M} 4=60$ days), who underwent were performed surgical fractures. In treated group, it was performed bone perforations on proximal and distal edges, craniolateral and mediolateral to the fracture site. At the end of each moment, control and treated animals were evaluated by radiography, histology, and bone mineral densitometry (BMD) was determined on fracture site. According to the radiographic data of treated dogs, it was verified on days 15 and 30 more intense bone regeneration than control group. During M3 and M4, it wasn't detected any difference in bone reparation process betweencontrol and treated groups. In densitometric study, BMD values were greater in treated animals than in control dogs. Histological studies revealed at 15 and 30 days chondrocyte hyperplasia and initial endochondral ossification on drilled limbs; control group showed sustainment connective tissue and initial chondrocyte hiperplasia. At M3 and M4 of the treated group, were verified development and remodeling of periosteal callus in more advanced phases when comparing with limbs from control group. It can be concluded that using perforations enhances blood flow supply and activation of osteogenic cells on fracture site, stimulating the beginning of fracture consolidation process.

\section{Referências}

1 EGER, C. E. A technique for the management of radial and ulnar fractures in miniature dogs using transfixation pins. JOURNAL SMALL ANIMAL PRACTICE, v. 31, p. 377-387, 1990.

2 EKMAN, B.; LJUNGQUIST, K. G.; STEIN, U. Roentgenologic photometric method for bone mineral determination. acta radiological diagnostic v. 10, p. $305,1970$.

3 LEAL, A. C. R. Determinação dos valores normais da densidade mineral óssea (DMO) da extremidade distal do rádio em cães por meio da técnica de densitometria óptica em imagens radiográficas: correlação entre o peso, sexo e idade. 2002. 51 f. Dissertação (Mestrado). Faculdade de Medicina Veterinária e Zootecnia, Universidade Estadual Paulista, Botucatu,2002.

4 PROPHET, E. B; MILLIS, B; ARRINGTON, J. B;
Key-words:

Fractures.

Bone drilling.

Densitometry.

Histology.

Dog.
SOBIN, L. H. Laboratory methods in histotechnology. Washington: Armed Forces Institute of Pathology, 1992.

5 RHINELANDER, L. M., BARAGRY, R. A. Microangiography in bone healing. I. Undisplaced closed fracture. JOURNAL BONE JOINT SURGERY: AMERICAN VOLUME, v. 44, p. 1273-1298, 1962.

6 SEEGER, L. L. Bone density determination. SPINE, v. 22, n. 245, p. 49s-57s, 1997.

7 VAUGHAN, L. C. A clinical study of nounion fracture in the dog. JOURNAL SMALL ANIMAL PRACTICE, v. 5, p. 173-77, 1964.

8 WELCH, J. A.; BOUDRIEAU, J. R.; DE JARDIN, L. M.; SPODNICK, J.G. The intraosseous blood supply of the canine radius: implication for healing of distal fracture in small dogs. VETERINARY SURGERY, v.26, p.57-61, 1997.

9 WILDEY, R.N. Un-united fracture by long axial drilling of the bone ends. BRITAIN JOURNAL 
SURGERY ASSOCIATION SURGERY: GREAT BRITAIN, p. 423-428, 1914.

10 SUMNER-SMITH, G. A histologycal study of fracture nounion in small dogs. J. Small Anim. Pract., v. 15, p. $571-578,1974$

11 WILLIAM, J. G. P. Recalcitrant stress fracture - a case managed by drilling. BRITAIN JOURNAL SPORTS MEDICINE, v. 13, p. 84-85, 1979.

12 MASSONE, F. Técnicas anestésicas em cães. In: MASSONE, F. Anestesiologia veterinária: farmacologia e técnicas. 2. ed. Rio de Janeiro: Guanabara Koogan, 1994. p 117-136.

13 BANKS, W. J. Tecidos de sustentação. In: BANKS, W. J. Histologia veterinária aplicada. 2. ed. São Paulo: Manole, 1992. p. 137-213.

14 SUMNER-SMITH, G.; CAWLEY, A. J. Nounion of fracture in the dog. JOURNAL SMALL ANIMAL PRACTICE, v. 11, p.311-325, 1970.

15 SPECH, T. E.; MILLER, G. J.; COLAHAN, P. T. Effect of clustered drill holes on the breaking strenght of the equine third metacarpal bone. AMERICAN JOURNAL VETERINARY RESEARCH, v. 51, p. 1242-1246. 1990.

16 WENTWORTH, R. A.; KALLFELZ, F. A. A.; HILTZ, F. L.; SCHRYVER, H.; SHEFFTY, B.; KROOK, L. P. In vivo estimations of bone mineral content. A research and diagnostic technique for veterinary medicine. AMERICAN JOURNAL VETERINARY RESEARCH, V. 32, p. 985, 1971

17 AL-HITI, J.; THOMAS, B. J.; AL-TIKRITY, S. A.; ETTINGER, K. V.; FREMLIN, J. D.; DABEK, J. T. Spinal calcium. It's in vivo measurement in man. international journal application radioative isotopes., v. 27, p. 97, 1976.

18 REMEDIOS, A. Bone and bone healing. VETERINARY CLINICS NORTH AMERICA: SMALL ANIMAL PRACTICE, v. 29, n. 5, p. 1029-1082, 1999.

19 GUEDEL, A. E. Inhalation anesthesia. 2. ed. New York: 1952. 250 p.

20 LOUZADA, M. J. Q.; BELANGERO, W. D.; PELÁ, C.A.; SANTOS-PINTO, R. Avaliação de densidade óssea em radiografias - II Estudo em cães. In: CONGRESSO BRASILEIRO DE ORTOPEDIA E TRAUMATOLOGIA, 30.,1996, Curitiba,PR. Anais... Curitiba, 1996.

21 LOUZADA, M. J. Q.; PAULIN, J. B. P.; XAVIER, C. A. M.; VALERI, V. Microdensitometria em radiografias de perfurações ósseas. REVISTA BRASILEIRA ORTOPEDIA, v. 24, n. 5, p. 165-168, 1989.
22 VULCANO, L. C. Determinação e padronização dos valores normais da densidade mineral óssea (DMO) do carpo acessório de eqüinos em crescimento da raça Puro Sangue Inglês (PSI), por meio da densitometria óptica em imagem radiográfica. 2001. ,52 f. Tese (LivreDocência). Faculdade de Medicina Veterinária e Zootecnia, Universidade Estadual Paulista, Botucatu,2001

23 MARKEL, M.; BOGDANSKE, J. Dual-energy X- ray absorptiometry of canine femurs with and without fracture fixation devices. AMERICAN JOURNAL VETERINARY RESEARCH, v. 55, p. 462-466, 1994.

24 RUDD, G. R.; WHITEHAIR, J. G. Fracture of the radius and ulna. VETERINARY CLINICS NORTH AMERICA: SMALL ANIMAL PRACTICE, v. 22, p. 13549, 1992.

25 WILKENS, B. E.; MILLIS, D. L.; DANIEL, G. B.; MUNSON, L.; PATEL, R. K.; BUONOMO, F. C. Metabolic and histologic effects of recombinat canine somatotropin on bone healing in dogs, using an unstable ostectomy gap model. AMERICAN JOURNAL VETERINARY RESEARCH, v. 57, n. 9, p. 1395-1401, 1996.

26 LOUZADA, M. J. Q. Otimização da técnica de densitometría óptica em imagens radiográficas de peças ósseas: estudo "In Vitro". 1994. 191 f. Tese (Doutorado) Faculdade de Engenharia Elétrica, Universidade Estadual de Campinas, Campinas, 1994

27 BEHMER, O. A.; TOLOSA, E. M. C.; FREITAS NETO, A. G. Manual de técnicas para histologia normal e patológica. São Paulo: EDART, 1976. 425 p.

28 DEANGELIS, P. M. Causes of delayed union and nounion of fracture. VETERINARY CLINICS NORTH AMERICA: SMALL ANIMALS PRACTICE, v. 5, p. 251 258, 1975.

29 LAPPIN, M.; ARON, D.; HERRON, H. L.; MALNATI, G. Fracture of the radius and ulna in the dog. JOURNAL AMERICAN ANIMAL HOSPITAL ASSOCIATION, v. 19, p. 643-650, 1983.

30 MILLIS, D.; WILKENS, B. E.; DANIEL, G. B.; HUBNER, K.; MATHEWS, A.; BUONOMO, C. F.; PATELL, K. R.; WEIGEL, J. P. Radiographic, densitometric, and biomechanical effects of recombinant canine somatotropin in an unstable ostectomy gap model of bone healing in dogs. VETERINARY SURGERY, v. 27, p. 85-93, 1998.

31 REICH, N. E.; SEIDLMANN, F. E.; TUBBS, R. R.; MACLNTYRE, W. J.; MEANY, T. F.; ALFIDIR, R. J.; PEPE, R. G. determination bone mineral content using 
CT scanning. AMERICAN JOURNAL

ROENTGENOLOGIC, v. 127, p. 593, 1976. 